testing performed by this laboratory corporation during June 2008-July 2010.

Results The dataset contained 3.26 million specimen records. Among those tested, $86.2 \%$ were women, $41.0 \%$ were aged 15-24 years, $73.5 \%$ had commercial insurance, $21.8 \%$ had Medicaid insurance, and $56.6 \%$ resided in the South. The most frequently used type of chlamydia test was a nucleic acid amplification test (77.5\%). Among women, $59.7 \%$ of specimens were cervical, $21.1 \%$ vaginal, and $18.8 \%$ urine. Overall, $4.0 \%$ of tests were positive. Positivity rates were highest in persons aged 15-19 years, and higher in men than women for all age groups. Rates also were higher in women with Medicaid insurance than women with private insurance.

Conclusions Systematically collected laboratory data can fill a critical gap in monitoring US chlamydia testing and positivity trends. These data are more representative of the US population by geographic distribution and insurance type than other data sources. The analysis of laboratory testing data might be useful for national surveillance that would not be dependent on provider or health department reporting. Our findings underscore the importance of screening young women for chlamydia, especially adolescents in whom screening rates are low. Men had higher positivity rates probably because they sought treatment for symptoms or were referred by an infected partner. Further analysis is needed to assess if testing of persons older than 25 years was according to guidelines, such as pregnant women, at-risk persons, or symptomatic persons.

\section{P1-S6.34 EVALUATION OF RISK-SCORE ALGORITHMS FOR THE DETECTION OF HIV INFECTION AND SYPHILIS IN NORTH CAROLINA COUNTY JAILS}

doi:10.1136/sextrans-2011-050108.258

${ }^{1} \mathrm{~L}$ A Sampson, ${ }^{2} \mathrm{~W}$ C Miller, ${ }^{1} \mathrm{P}$ A Leone. ${ }^{1}$ NC Division of Public Health, Raleigh, USA; ${ }^{2}$ University of North Carolina, Chapel Hill, USA

Background In North Carolina, the funding environment for jail screening has shifted from a syphilis-centred model to one based on integrating HIV and STI testing. We developed and evaluated risk score algorithms for HIV screening with and without the addition of syphilis screening in North Carolina county jails.

Methods This study included 3610 inmates screened for both syphilis and HIV in two North Carolina jails, 2002-2005. We verified reactive syphilis tests against surveillance records to identify new syphilis cases. We created logistic regression models to predict two different outcomes: HIV only and HIV or syphilis. We created risk scores by rounding the $\beta$ coefficients from the final models. Cut-offs were chosen based on testing $50 \%$ of the available inmate population. We calculated the sensitivity and specificity for each of the risk scores for three outcomes: HIV only, syphilis only, HIV or syphilis. Analyses were conducted using Stata.

Results The final model for the HIV-only outcome included sex, age, ever tested for HIV, and race/ethnicity. The lowest scoring individual type would be a heterosexual man, age 18-24, never tested for HIV, with a race/ethnicity in the referent group (total score $=0$ ). The highest scoring individual would be MSM, age 25 or older, previously tested for HIV, and of Hispanic or Black nonHispanic race/ethnicity (total score=6). A risk score cutoff of three or above will lead to screening $<50 \%$ of the available inmate population. The weighted risk scores from the HIV-only outcome model had better sensitivity for the detection of HIV (82.6, 95\% CI 71.2-to 94.0) than the combined-outcome model (65.2 95\% CI 50.9-to 79.5). If inmates are selected for screening based on the HIV model, the sensitivity for detection of new syphilis cases is also good (73.3, 95\% CI 56.5-to 90.1) and is only slightly inferior to the HIV or syphilis model $(80.095 \% \mathrm{CI}$ 64.8-to 95.2).

Conclusions We believe that the screening algorithm will perform well in the county from which the sample was drawn. Generalisation to other communities in the Southern USA with similar demographics and rates of HIV and syphilis is also possible. More generally, we recommend targeting HIV jail screening efforts based on HIV data. In communities with incident syphilis infections, we recommend adding syphilis screening to the HIV protocol.

\section{P1-S6.35 ONE FINGER STICK, TWO TESTS: INTEGRATING SYPHILIS AND HIV RAPID TESTS FOR ANTENATAL CARE AND REPRODUCTIVE HEALTH SERVICES}

doi:10.1136/sextrans-2011-050108.259

M Valderrama, M Chiappe. Universidad Peruana Cayetano Heredia, Lima, Peru

Background Mother-to-Child Transmission of HIV and Congenital Syphilis are two important public health problems in many developing countries. In Peru, coverage of syphilis screening is suboptimal, test results can take weeks and a high proportion of women screened are not treated, much less their sex partners. The objective of this study was to demonstrate the feasibility of implementing syphilis and HIV rapid tests at antenatal care and reproductive health services and to measure its effect on coverage of maternal syphilis screening and treatment coverage.

Methods We trained midwives from 16 health centers, to offer syphilis rapid tests (Syphilis 3.0 BioLine) to women 16 to 55 years old receiving antenatal care (ANC) and seen at the delivery or miscarriage services, and to integrate it with HIV rapid tests already in place at the health network, but which were until then done from venous blood by laboratory technicians and with results given in 15 to 30 days.

Results 4497 women were screened, from January to November 2010. Prevalence of syphilis (RPR reactive, TPPA $(+)$ ) was $0.9 \%$ in ANC, $0.7 \%$ in delivery services and $1.9 \%$ at the miscarriage services. The prevalence of HIV by rapid test was $0.3 \%$. The overall coverage for syphilis and HIV rapid tests was $93 \%$ during the study, compared to the $35 \%$ coverage of RPR testing reported for 2009 (Abstract P1-S6.35 figure 1). Previous coverage of HIV testing was not documented. Test results were given to $100 \%$ of women within 20 min of finger stick blood collection during the first contact with health providers, compared to a 27 days delay found in 2009. Treatment rates for syphilis also improved from $51 \%$ in 2009 to $93 \%$ in 2010 . Whereas previously there was no system in place for registering partner treatment, our data shows that $59 \%$ of partners of positive women received treatment for syphilis during the study period. Key factors in the success of implementation in Callao-Peru; were ongoing training, monitoring, and supervision of health providers who performed the rapid tests. 


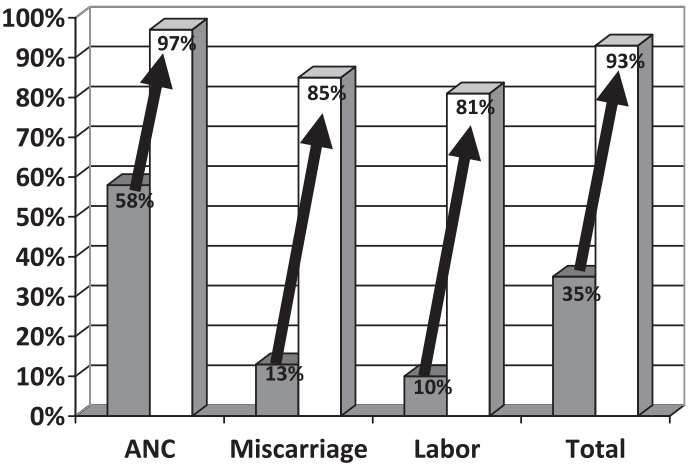

$\square 2009$ (RPR screening) $\square 2010$ (PRS screening)

Abstract P1-S6.35 Figure 1 Coverage of Syphilis screening by year and service Callao, Peru 2009-2010.

Conclusions Implementation of syphilis rapid test proved feasible, acceptable and effective in improving screening and treatment coverage and can serve as a powerful catalyst for improvements in quality of care.

\section{P1-S6.36 COST-EFFECTIVENESS OF RAPID TESTS TO IMPROVE ACCESS TO SYPHILIS PREVENTION AMIONG PREGNANT WOMEN IN PERU}

doi:10.1136/sextrans-2011-050108.260

${ }^{1} \mathrm{P}$ Mallma, ${ }^{1} \mathrm{C}$ Carcamo, ${ }^{1} \mathrm{M}$ Valderrama, ${ }^{1} \mathrm{M}$ Chiappe, ${ }^{1} \mathrm{~S}$ La Rosa, ${ }^{1} \mathrm{P}$ Garcia, ${ }^{2} \mathrm{M}$ Weaver, ${ }^{2} \mathrm{~K}$ Holmes, ${ }^{3} \mathrm{~F}$ Terris-Prestholt, ${ }^{3}$ Rossana Peeling. ${ }^{1}$ School of Public Health, Universidad Peruana Cayetano Heredia, Lima, Peru, ${ }^{2}$ University of Washington, Seattle, USA; ${ }^{3}$ London School of Hygiene and Tropical Medicine, London, UK

Background Several recent articles compared the cost and costeffectiveness syphilis testing strategies to avert congenital syphilis in settings with high syphilis prevalence. Current study contributes analysis in low-prevalence setting. Methods. Rapid syphilis testing (RST) was implemented at two different settings, both with syphilis prevalences of around 1\%: (a) The Ventanilla-Network of outpatient clinics and a small hospital at a peripheral district in Peru, where the rapid syphilis test was implemented together with the rapid HIV testing (One finger stick, two tests"); (b) The National Maternal and Perinatal Institute (INMP) a tertiary hospital with a high number of patients, and Initially in both settings the only test available was RPR with low coverage. For the costing of RPR we included supplies, capital costs, human resources and costs associated to treatment. For the costing of RST, we included also start up costs (advocacy meetings with authorities, training, supervision, monitoring) and the cost of implementing a quality assurance system. Cost-effectiveness analyses compared the cost of screening and treatment for the joint program to the disability adjusted lifeyears saved when congenital syphilis was averted.

Results For the Ventanilla-Network the total cost was $\$ 5.98$ for RST and $\$ 5.22$ for RPR per woman screened and $\$ 580.83$ and $\$ 1845.55$ respectively per woman treated. In contrast, the total cost was $\$ 2.53$ for rapid syphilis test and $\$ 3.15$ for RPR per woman screened (the lower costs probably associated to the economy of scale, due to the large number of women seen at the INMP) and $\$ 336.80$ and $\$ 1051.59$ at INMP. At Ventanilla-Network, the cost per DALY saved from averting cases of congenital syphilis was $\$ 35.23$ for rapid syphilis test and $\$ 111.95$ for RPR. In incremental analysis, the rapid test was cost-saving. At INMP, the cost per DALY saved was $\$ 20.43$ for rapid syphilis test and $\$ 63.79$. For the Ventanilla-Network and the INMP the RST was cost-effective by the WHO standard of \$64/ DALY.

Conclusion Syphilis screening is cost-effective even in a low-prevalence setting. To the extent that HIV rapid tests are funded by PMTCT programs, the cost of scaling up rapid syphilis tests would be lower than these estimates, because the joint cost of blood sample collection would be borne by the PMTCT program.

\section{P1-S6.37 CAN RAPID SYPHILIS TESTS BE IMPLEMENTED AND IMPROVE SCREENING FOR MATERNAL SYPHILIS IN A THIRD LEVEL HOSPITAL?}

doi:10.1136/sextrans-2011-050108.261

S La Rosa Roca, P Garcia. Cayetano Heredia University, Lima, Peru

Background Syphilis continues to be one of the most important causes of maternal and child morbi-mortality, frequently being more common than HIV. Ideal diagnostic test should provide rapid and accurate results to provide timely diagnosis and treatment. Rapid syphilis testing is an excellent option. The objective of this study is to determine the feasibility of the implementation of Rapid Syphilis Testing (RST, Syphilis 3.0 BioLine) and the impact on maternal screening for syphilis at a third level hospital in Peru: the National Maternal and Perinatal Institute (INMP).

Methods Between February and December of 2010, RST was implemented in the Antenatal care (ANC), labour and miscarriage services. The National guidelines recommended screening for syphilis in all those services with RPR. Health workers (midwives, nurses and laboratory technicians, depending on the service) were properly trained on the use of RST and their performance was monitored throughout the study.

Results RST was performed on 15116 women, with a prevalence of syphilis (RPR reactive, TPPA positive) of $1 \%$ for ANC, $0.7 \%$ for miscarriage services and $0.8 \%$ at labour. The coverage of screening for syphilis improved from $82 \%$ with RPR to $99 \%$ with RST at the ANC services; from $0 \%$ to $91 \%$ at the miscarriage services and from $79 \%$ to $94 \%$ at labour. At baseline, at the ANC visit, the results of the RPR were only available 15 days after the visit, resulting in a low coverage for treatment when the test was positive, 39\%. With the implementation of the RST, the results and treatment were given immediately at the same visit, resulting on treatment coverage of $95 \%$. The RST was very well accepted by the providers and patients.

Conclusions This study shows the feasibility, acceptability and improvements on screening associated to the implementation of RST in a third level hospital. In addition of improving coverage and treatment, our results triggered changes in the institutional policies for syphilis control.

\section{P1-S6.38 INTERPRETATION OF ROUTINE DATA FROM A YOUTH FRIENDLY CLINIC IN REGION F, JOHANNESBURG}

doi:10.1136/sextrans-2011-050108.262

S T Lalla-Edward. Wits Institute for Sexual and Reproductive Health, HIV and related diseases, Johannesburg, South Africa

Background The Wits Institute for Sexual and Reproductive Health, HIV and Related Diseases (WrHI)'s Youth Friendly Programme aims to engage with young people and make reproductive healthcare accessible and non-threatening. The team educates and informs young people about HIV and has specially sensitised nurses and 